\section{Gratulation an Professor Dr. Dietrich Berdel}

\author{
Manchmal gibt es Gründe für eine Laudatio auch außerhalb runder \\ Geburtstage: So feierte Prof. Dr. med. Dietrich Berdel dieses Jahr am \\ 22. Februar „nur“ seinen 64. Geburtstag, er hat aber zum Jahres- \\ wechsel 2008/2009 die von ihm in den vergangenen 20 Jahren auf- \\ gebaute Kinderklinik des Marien-Hospitals in Wesel in jüngere Hände \\ übergeben und damit ein wichtiges Beispiel gegeben.
}

D ie Kinderklinik Wesel ist in den letzten 20 Jahren zu einem Zentrum der klinischen und wissenschaftlichen pädiatrischen Allergologie im Westen unserer Republik geworden. Dieser Erfolg kann als Musterbeispiel und Anregung für jüngere Kollegen dienen. Kompetente Wissenschaft lässt sich auch außerhalb der universitären Zentren etablieren. Es ist sogar zu erwarten, dass dieses Beispiel einer schlagkräftigen dezentralen Einrichtung unter geeigneter Leitung - und mit der Mitwirkung einer kooperativen Verwaltung - für die $\mathrm{Zu}$ kunft wegweisende Impulse geben kann in dem Maße, in dem universitäre Strukturen immer komplizierter, schwerfälliger und aus Kostengründen auch wissenschaftsfeindlicher werden.

\section{Von Niedersachsen an den Niederrhein}

Dietrich Berdel ist am 22. Februar 1945 in Sulingen geboren. Seine frühe Kindheit verbrachte er in Jesteburg und Wohltorf. Er besuchte das Gymnasium des Landschulheims am Solling in Holzminden. Das Medizinstudium absolvierte er von 1965 bis 1971 in Innsbruck und in Homburg/Saar. Die Medizinalassistentenzeit durchlief er am Städtischen Krankenhaus in Offenburg und an der Virologischen Abteilung des Institutes für Hygiene und Mikrobiologie der Universität Homburg/Saar. Die pädiatrische Weiterbildung begann Dietrich Berdel 1972 in Homburg/Saar. Er setzte die Weiterbildung nach Ableistung der Wehrpflicht an der Universitäts-Kinderklinik in Bonn fort. Dort war er im Anschluss bis zum 30. Juni 1988 tätig, seit dem 19. Dezember 1979 als Oberarzt

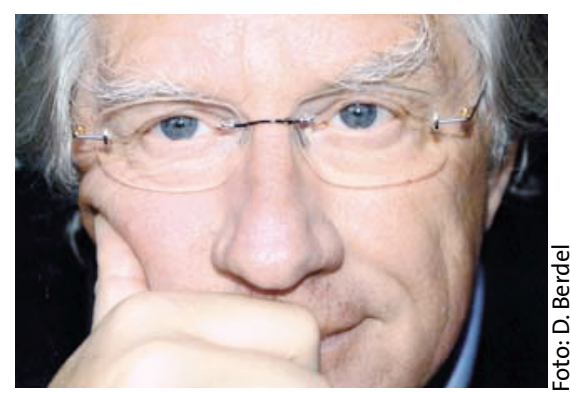

Ad multos annos: Prof. Dr. Dietrich Berdel

und seit dem 13. Juli 1984 als Professor (C2) auf Zeit. Am 22. November 1988 wurde ihm die Bezeichnung außerplanmäßiger Professor verliehen.

Am 1. Juli 1988 wurde Dietrich Berdel Chefarzt der Kinderabteilung des Marien-Hospitals zu Wesel, von 1995 bis 2007 war er außerdem Ärztlicher Direktor der Klinik.

\section{Schwerpunkt pädiatrische Pneumologie und Allergologie}

Die pädiatrische Pneumologie und Allergologie ist der Mittelpunkt der wissenschaftlichen Arbeit von Dietrich Berdel. Dies wird auch nach seinem Ausscheiden aus der Chefarztposition sein Schwerpunkt in dem von ihm begründeten wissenschaftlichen Institut an der Kinderklinik Wesel bleiben. Zu den Hauptthemen der Forschung gehören diagnostische Methoden im Kleinkindesalter und die Besonderheiten der Therapie mit Antiasthmatika im Kindesalter.

Bei allem eigenen Engagement sind immer auch die Befähigung und die Bereitschaft zum Einsatz der Mitarbeiter für den Erfolg von Chefärzten von größter Bedeutung. Hier war Dietrich
Berdel in besonderem Maße von Glück gesegnet. Durch die Zusammenarbeit mit seiner Frau, Dr. med. Andrea von Berg, wurde der wissenschaftliche Erfolg der Kinderklinik Wesel vollendet, vor allem durch die erhebliche Mittelzuwendung auf dem Gebiet der Allergieprävention. Auf diesem Gebiet haben Dietrich Berdel und Andrea von Berg in den letzten Jahren mit hohen Impactfaktoren publizieren können, nach langjährigen, multizentrischen, von Wesel aus organisierten Studien zur Ernährung im Säuglingsalter.

\section{Einsatz in zahlreichen Arbeitskreisen}

Neben der Tätigkeit als Chefarzt und Ärztlicher Direktor sowie als Organisator wissenschaftlicher Studien hat Dietrich Berdel weitere Aufgaben übernommen. Hierzu gehören:

- die federführende Mitarbeit in der Arbeitsgemeinschaft der Gesellschaft für Pädiatrische Pneumologie (GPP) für die Asthmatherapie,

— die Mitarbeit als Vertreter der GPP im Gemeinsamen Bundesausschuss für das Disease-Management-Programm Asthma,

- die Mitarbeit als Vertreter der GPP und der Gesellschaft für Pädiatrische Allergologie und Umweltmedizin (GPA) an der Zentralstelle der Deutschen Ärzteschaft zur Qualitätssicherung in der Medizin für die Nationalen Versorgungsleitlinien Asthma bronchiale.

Jeder, der sich in derartigen Arbeitskreisen engagiert hat, weiß, welcher Einsatz bei diesen Aufgaben erwartet wird - ohne jegliche Kompensation. Das Arbeitszeitgesetz gilt bekanntlich nicht für Chefärzte. Wenn er dann in derartigen Gremien seine immer begründete Meinung gelegentlich auch - angesichts anderer weniger sinnvoller Statements - überdeutlich mitgeteilt hat, dann gebührt ihm hierfür jegliches Verständnis.

Dietrich Berdel ist zu wünschen, dass ihm nunmehr für sein Engagement in der pädiatrischen Forschung ebenso die notwendige Zeit zur Verfügung stehen wird wie für seine erste Heimat am Niederrhein und sein zweite Heimat in Griechenland.

Prof. Dr. Gerhard Schultze-Werninghaus, Bochum 